\title{
Geant4 interaction model comparison for dose deposition from gold nanoparticles under proton irradiation
}

DOI:

10.1088/2057-1976/aa69cc

\section{Document Version}

Accepted author manuscript

Link to publication record in Manchester Research Explorer

\section{Citation for published version (APA):}

Sotiropoulos, M., Taylor, M., Henthorn, N., Warmenhoven, J., Mackay, R. I., Kirkby, KJ., \& Merchant, M. (2017). Geant4 interaction model comparison for dose deposition from gold nanoparticles under proton irradiation. Biomedical Physics \& Engineering Express, 3, [025025]. https://doi.org/10.1088/2057-1976/aa69cc

\section{Published in:}

Biomedical Physics \& Engineering Express

\section{Citing this paper}

Please note that where the full-text provided on Manchester Research Explorer is the Author Accepted Manuscript or Proof version this may differ from the final Published version. If citing, it is advised that you check and use the publisher's definitive version.

\section{General rights}

Copyright and moral rights for the publications made accessible in the Research Explorer are retained by the authors and/or other copyright owners and it is a condition of accessing publications that users recognise and abide by the legal requirements associated with these rights.

\section{Takedown policy}

If you believe that this document breaches copyright please refer to the University of Manchester's Takedown Procedures [http://man.ac.uk/04Y6Bo] or contact uml.scholarlycommunications@manchester.ac.uk providing relevant details, so we can investigate your claim.

\section{OPEN ACCESS}




\title{
Geant4 interaction model comparison for dose deposition from gold nanoparticles under proton irradiation
}

\author{
M Sotiropoulos ${ }^{1}$, M J Taylor ${ }^{1}$, N T Henthorn ${ }^{1}$, J W Warmenhoven ${ }^{1}$, R I \\ Mackay $^{2}$, K J Kirkby ${ }^{1,3}$, and M J Merchant ${ }^{1,3}$ \\ ${ }^{1}$ Division of Molecular and Clinical Cancer Sciences, Faculty of Biology, Medicine and \\ Health, The University of Manchester, UK \\ ${ }^{2}$ Christie Medical Physics and Engineering, The Christie NHS Foundation Trust, \\ Manchester, UK \\ ${ }^{3}$ The Christie NHS Foundation Trust, Manchester, UK \\ E-mail: marios.sotiropoulos@ics.manchester.ac.uk
}

\begin{abstract}
Gold nanoparticles (GNPs) have shown a potential as a radiosensitizer in radiotherapy. The radiosensitization effect is thought to be linked to the increased dose deposition around the GNP. Monte Carlo simulations have been implemented for the calculation of the dose distributions around the GNPs and have been used for the calculation of the dose enhancement. They have also been imported to radiobiological models to predict biological endpoints. This work assessed the implications of different physical interaction models on the dose distribution and dose enhancement of GNPs surrounded by water under proton irradiation. The Penelope and Livermore physical interaction model implementation of the Geant4 simulation toolkit were compared considering the following parameters: i) GNP size, ii) proton energy, and iii) alternative physics model parameters in the gold or water medium. We found that neither the dose distribution nor the dose enhancement is sensitive to the model selection after the first $100 \mathrm{~nm}$ from the GNP surface. Within the first $100 \mathrm{~nm}$ the Livermore models calculated a higher dose, attributed to a higher production of low energy secondary electrons inside the GNP.
\end{abstract}

Keywords: Gold Nanoparticles, radio-sensitizer, Geant4-DNA, Proton Beam

\section{Introduction}

The ultimate goal of radiation therapy is to deliver high radiation dose to the tumour, while sparing the normal tissues and organs at risk. Usually the dose in the tumour is constrained by the prescribed limits to dose in the normal tissues including organs at risk, since high dose to normal tissues is linked to increased treatment side effects. Therefore, new methods are under investigation in order to boost the dose at the tumour site, while sparing the adjacent tissues. Gold nanoparticles (GNPs) have demonstrated radiosensitization potential in vivo and in vitro, under irradiation with $\mathrm{x}$-rays, electrons, and protons (Hainfeld et al 2004, Liu et al 2010, Polf et al 2011, Kim et al 2012).

While the radiosensitization effect may also be attributed to biological or chemical mechanisms, the physical interaction of the radiation with the GNPs is the main contributing factor to the effect through the production of secondary radiation. This is due to the production of low energy secondary radiation which deposits energy close to the GNP, enhancing the local dose. Monte Carlo (MC) simulations for the quantification of the local dose enhancement have been implemented by many groups (McMahon et al 2011b, Leung et al 2011, Wälzlein et al 2014, Lin et al 2014, Xie et al 2015). 
As each MC code utilizes different interaction models, a wide range on the dose values has been reported. For instance, in the case of x-ray irradiation Li et al. (2014) reported a dose enhancement value difference of a factor of 2 between the Penelope-2011 (Salvat et al 2011) and Geant 4 code (Agostinelli et al 2003, Allison et al 2006). Moreover, dose distributions around the GNPs have been used in combination with radiobiological models (Lechtman et al 2013, Lin et al 2015, McQuaid et al 2016) in order to calculate biological endpoints such as cell survival.

This interest in the nanoscale simulations raises a fundamental question; whether the physical interaction model selection can affect the predicted dose distribution around the GNP. Although different models for the interaction of protons with gold are provided in various MC codes for the simulation of protons with gold, we have selected to limit our study to one code that provides two different interaction models. Doing so will allow us to examine the differences due to the physical interaction models which otherwise may be masked by differences between codes. Therefore, we are investigating the effect of the interaction model selection on GNPs under proton irradiation for one of the most widely used Monte Carlo codes for nanoscale simulations, the Geant 4 simulation toolkit.

\section{Materials and Methods}

\subsection{Monte Carlo simulations}

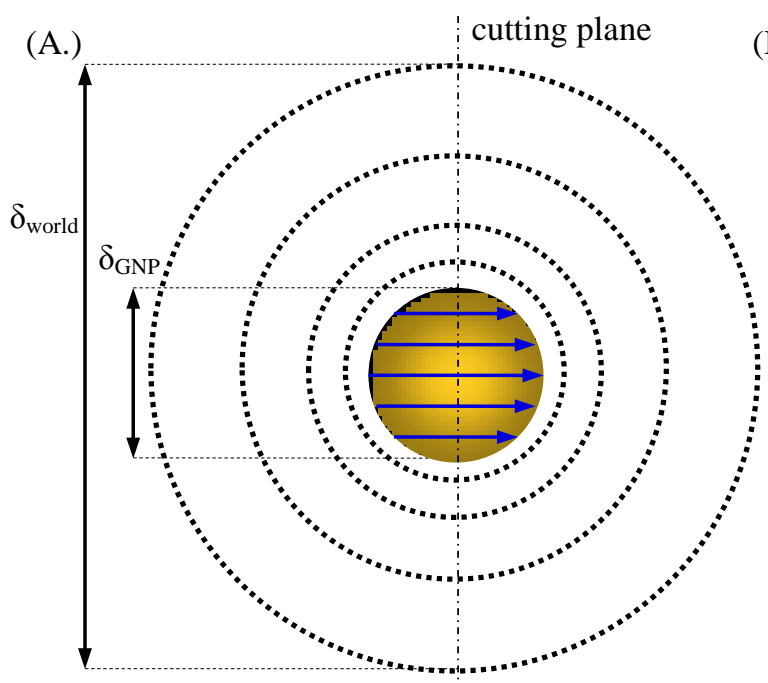

(B.)

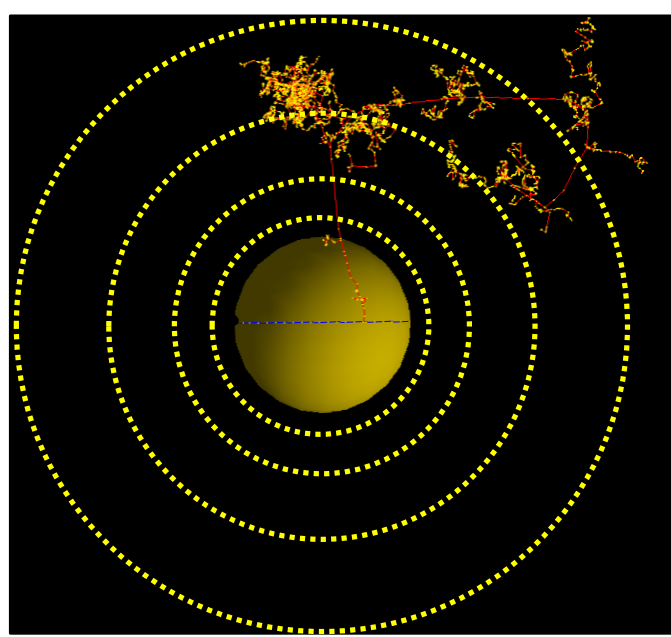

Figure 1. A.) The irradiation Geometry: The GNP is irradiated by a parallel proton beam (blue) starting and ending at the GNP inner surface. B.) The proton irradiation results in the production of secondary electrons (red). The scoring spherical shells are shown as dashed lines, with the vertical line separating the shells to hemispheres.

The Geant 4 v10.2 simulation toolkit was adopted for the calculation of the dose deposition around the GNP. In Geant 4 a physical interaction is described by a "process" which can combine multiple physics "models", depending on the region of the defined geometry and energy range. A collection of processes and corresponding models is referred as the "Physics List". Two "Physics Lists", namely "Livermore" and "Penelope" (Geant4 Collaboration 2015), are appropriate for the interactions of protons with the GNP. These "Physics Lists" allow the tracking of photons, electrons, positrons and protons. Both "Physics Lists" implement the same proton interaction models, while the photon, electron, and positron models are "Physics List" specific. The recommended applicability limit for electrons in "Livermore Physics List" is $250 \mathrm{eV}$, but can be used down to $10 \mathrm{eV}$ with reduced accuracy. For the "Penelope Physics List" the applicability limit is $100 \mathrm{eV}$. In the framework of the 
Geant4-DNA project (Incerti et al 2010, Bernal et al 2015), an alternative "Physics List" is available for liquid water simulating the dominant physical interactions of electrons down to thermalization energy $(\sim 25 \mathrm{meV})$.

For this study we combine either the "Livermore" or "Penelope" "Physics List" for the GNP with the Geant4-DNA "Physics List" for the surrounding water. In the reference scenario electrons, positrons, and protons are simulated, with the production threshold for electrons set to $10 \mathrm{eV}$ for the "Livermore/Geant4-DNA" or $250 \mathrm{eV}$ for the "Penelope/Geant4-DNA" combination. The influence of other production threshold values is also investigated.

The "Livermore" and "Penelope" "Physics Lists" incorporate the so called condensed history $(\mathrm{CH})$ algorithms for the calculating of the energy loss of the charged particles. In this formalism the interactions of the charged particle are not calculated in an event-by-event fashion, but the cumulative effect of a number of interactions is considered. The simulation of a single particle track is broken down into a series of smaller segments called steps. For the Multiple Coulomb Scattering the default "Urban" model is being used in both cases.

The improved de-excitation models introduced in the 10.2 version of Geant4 (Incerti et al 2016) are activated in order to simulate fluorescence, Auger electron production, and particle induced x-ray emission (PIXE).

The "Physics Lists" and models used are summarized in Table 1. In the text we keep the term "Physics List" to refer to the default model combination provided by Geant 4 and we will use the term "Mixed Physics List" to emphasize the "Physics List" combining "Livermore" or "Penelope" models with Geant4-DNA models. For instance, "Livermore Physics List" is referred to the Geant4 default "Physics List". On the other hand the term "Livermore Mixed Physics List" means the combination of the "Livermore Physics List" for the nanoparticle and the Geant4-DNA "Physics List" for the surrounding water material.

Table 1. "Physics List" combinations (i.e. "Mixed Physics Lists") used in the comparison for the proton interactions with the gold (GNP) or water nanoparticle (WNP), and surrounding water.

\begin{tabular}{|c|c|c|c|c|}
\hline & GNP & WNP & Water & denoted as \\
\hline \multirow{5}{*}{ 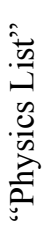 } & \multicolumn{2}{|c|}{ Livermore } & Geant4-DNA default & Livermore \\
\hline & \multicolumn{2}{|c|}{ Livermore, Auger Off } & Geant4-DNA default & AugerOff \\
\hline & \multicolumn{2}{|c|}{ Livermore } & Geant4-DNA/Emfietzoglou* & Emfietzoglou \\
\hline & \multicolumn{2}{|c|}{ Penelope } & Geant4-DNA default & Penelope \\
\hline & N/A & Geant4-DNA default & Geant4-DNA default & DNA default \\
\hline
\end{tabular}

\subsection{Irradiation geometry}

A single GNP with the radii of $1.0,7.5$, or $25.0 \mathrm{~nm}$ immersed in a water sphere representing the surrounding water environment with the radius of $1 \mu \mathrm{m}$ was simulated. The GNP was irradiated with 3,25 , or $100 \mathrm{MeV}$ protons instantiated from the inner half-surface of the sphere defining the GNP as depicted in Figure 1. The dose deposition was scored between the GNP surface and the water outer boundary in spherical shells with logarithmic thickness, forming the Radial Dose Distribution (RDD). Also, the dose deposited to two hemispherical shells formed by cutting the spherical shells perpendicular to the beam direction was scored.

Similarly to the GNP irradiations a control case of an equivalent water nanoparticle (WNP) was irradiated in order to calculate the Dose Enhancement Ratio (DER) in each case. The DER is defined as the ratio of the dose deposited from a GNP to that of a hypothetical WNP, and it is an index of the 
local dose enhancement. In all cases enough particles were simulated to ensure mean statistical uncertainty less than $2 \%$.

The protons only cross the water or gold nanoparticles volume and are "killed" outside of this NP volume. The energy deposited in the water volume surrounding the nanoparticles is therefore solely from the electrons produced inside the nanoparticles volume.

\section{Results}

The case of a single gold or water nanoparticle surrounded by water is simulated. The influence of the secondary production threshold, GNP size, proton energy, model selection on the calculated RDD and DER, and directionality of the RDD and DER are under investigation.

3.1. Dependence of the dose distribution and dose enhancement on the secondary production threshold
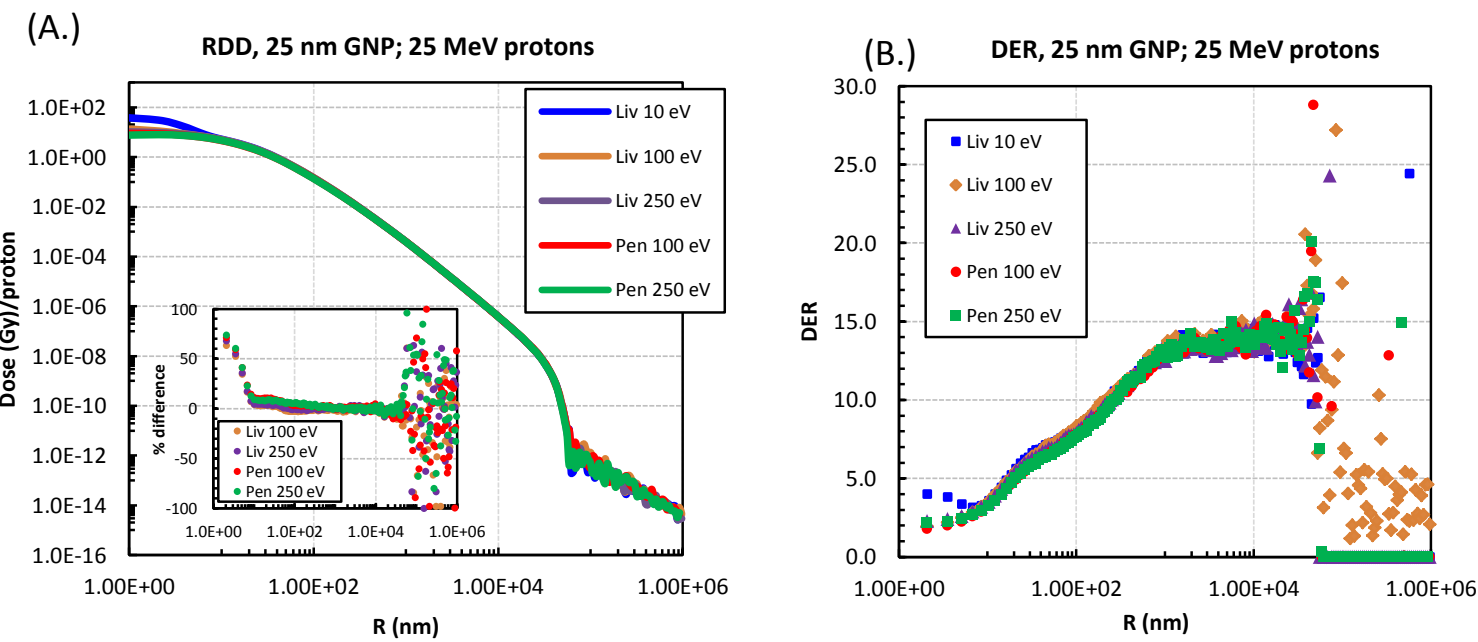

Figure 2. (A.) RDD and (B.) the DER for a GNP with a radius of $25.0 \mathrm{~nm}$, irradiated with $25 \mathrm{MeV}$ protons. Either the "Livermore" with the cut-off of 10,100, $250 \mathrm{eV}$ or "Penelope Mixed Physics List" with the cut-off of 100, and $250 \mathrm{eV}$ is being used, denoted as Liv and Pen respectively. The dose percentage difference from the "Livermore Mixed Physics List" with $10 \mathrm{eV}$ cut-off is presented.

Figure 2 shows the impact of the cut-off threshold to the RDD (A.) and the DER (B.) for a $25.0 \mathrm{~nm}$ GNP irradiated with $25 \mathrm{MeV}$ protons incorporating the "Livermore" or "Penelope" "Mixed Physics List". In the range $100 \mathrm{~nm}-30 \mu \mathrm{m}$ an agreement of less than $3 \%$ is observed for the RDD, with respect to the "Livermore Mixed Physics List" - $10 \mathrm{eV}$ cut-off. In Figure 3 the spectra of the electrons escaping the GNP and WNP of the previous scenarios, as well as the spectrum generated from the Geant4-DNA "Physics List" are also presented. For the following sections we focus on the "Livermore" - 10 eV cut-off and "Penelope" - 250 eV cut-off "Mixed Physics List". 

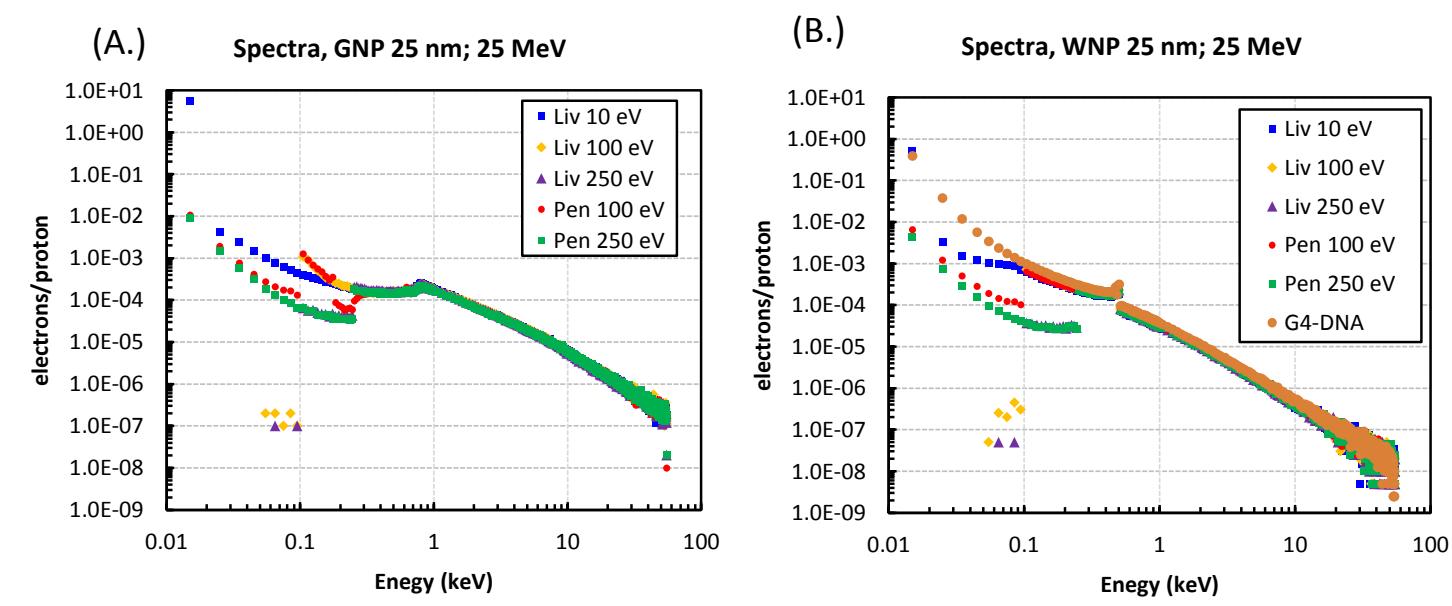

Figure 3. Spectra of the escaping electrons from the (A.) GNP and (B.) WNP. Either the "Livermore" with the cut-off of 10, 100, $250 \mathrm{eV}$ or "Penelope" "Mixed Physics List" with the cutoff of 100, and $250 \mathrm{eV}$ is being used. In the case of the WNP results from the Geant4-DNA "Physics List" are also presented.

\subsection{Dependence of the dose distribution and dose enhancement on the GNP size}

Figure 4 shows the RDD (A.) and the DER (B.) for a GNP irradiated with $25 \mathrm{MeV}$ protons incorporating either the "Livermore" or "Penelope" "Mixed Physics List". Three GNP radii were considered; i.e. 1, 7.5, and $25 \mathrm{~nm}$. RDDs are normalized per initial proton crossing the GNP. The RDDs for all the GNP sizes are very similar and no difference from the model choice is observed after the first $100 \mathrm{~nm}$. DERs are not affected by the "(Mixed) Physics List"; after the first $100 \mathrm{~nm}$ the DERs are similar and equal to about 14, until the maximum range of the secondary electrons. The maximum energy transferred to a free electron by a $25 \mathrm{MeV}$ proton is calculated to be $\sim 50 \mathrm{keV}$, corresponding to a maximum range of $\sim 40 \mu \mathrm{m}$. After that distance there is not any contribution to the dose from the electrons produced inside the GNP and the RDD decreases sharply. 
(A.)

RDD, $25 \mathrm{MeV}$ protons

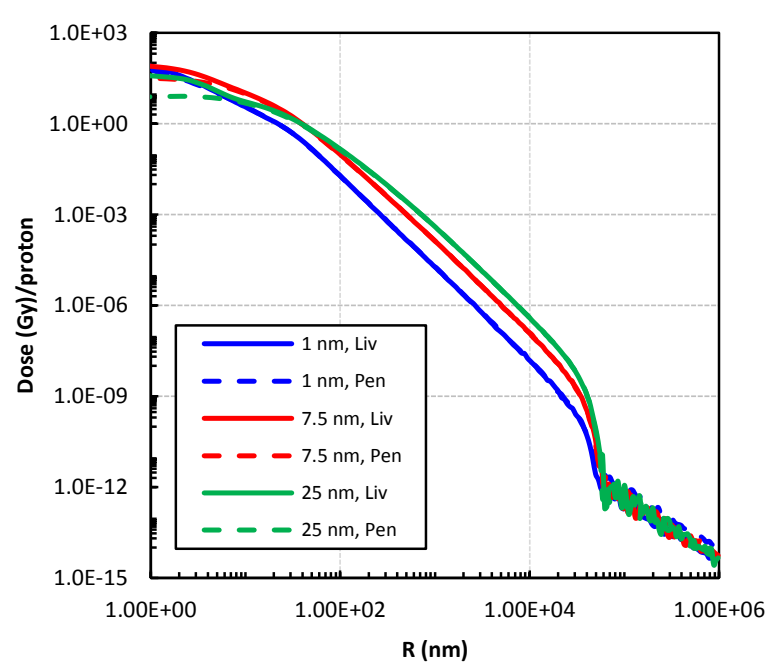

(B.)

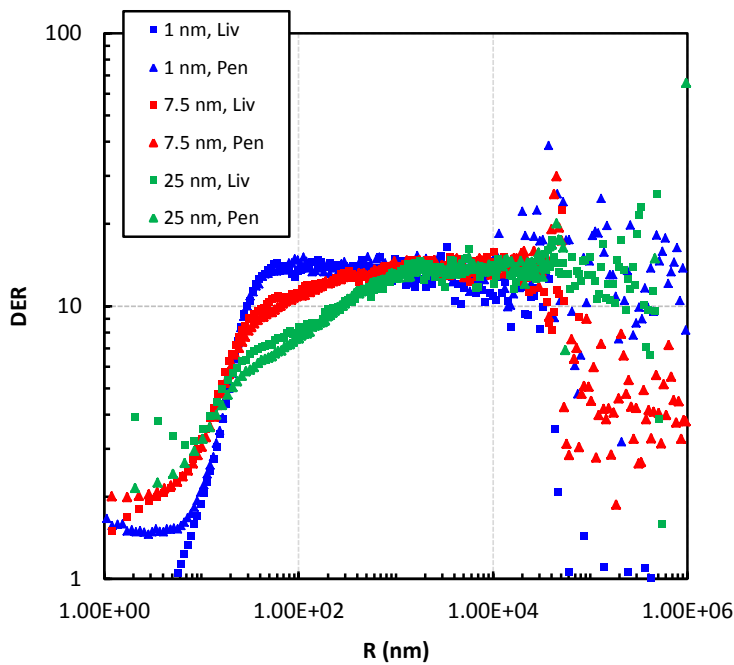

Figure 4. (A.) RDD and (B.) the DER for a GNP with a radius of 1.0, 7.5, or $25.0 \mathrm{~nm}$, irradiated with $25 \mathrm{MeV}$ protons. Either the "Livermore" or "Penelope Mixed Physics List" is being used denoted as Liv and Pen respectively.

\subsection{Dependence of the dose distribution and dose enhancement on the proton energy}

Figure 5 demonstrates the energy dependence in the "(Mixed) Physics List", by comparing the (A.) RDD and (B.) the DER for a $25 \mathrm{~nm}$ radius GNP, irradiated with 3, 25, and $100 \mathrm{MeV}$ protons. Neither the RDD nor the DER is affected by the "(Mixed) Physics List". The RDD and DER are independent of the energy for distances ranging from around $20 \mathrm{~nm}$ to the maximum secondary electron range and reaches the value of 14 after the first $200 \mathrm{~nm}$.

(A.)

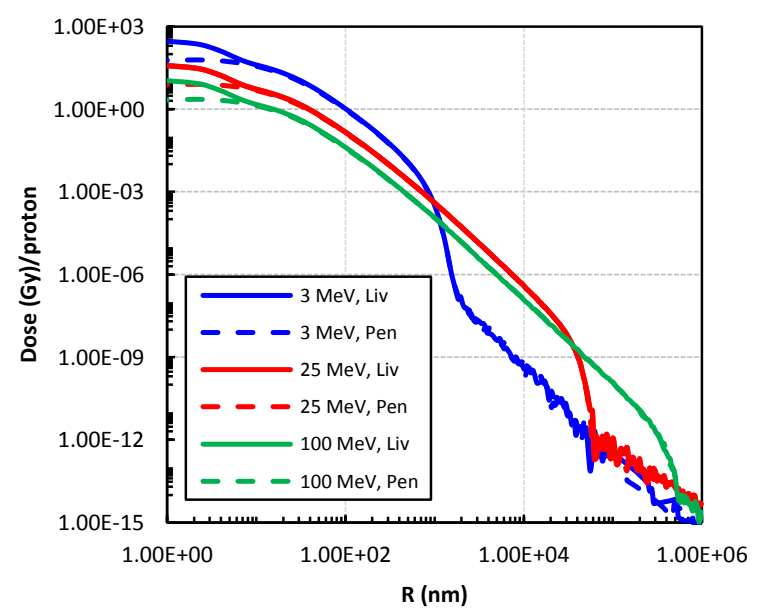

(B.)

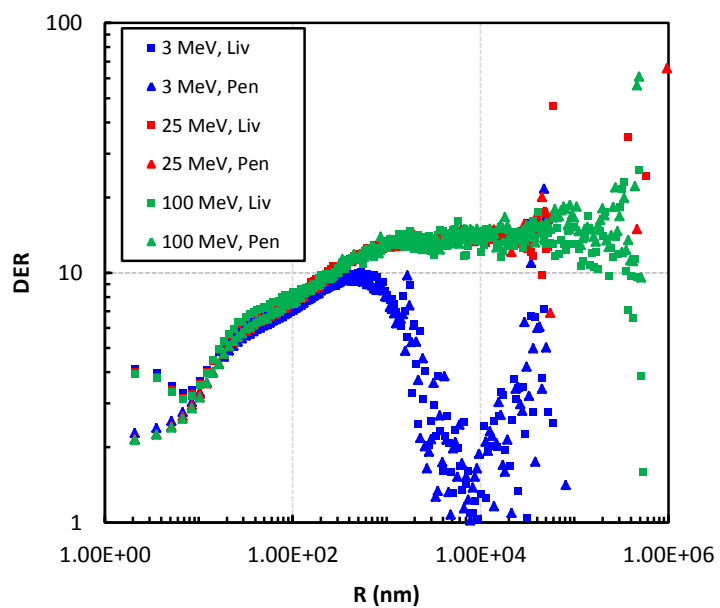

Figure 5. (A.) RDD and (B.) the DER for a GNP with a radius of $25 \mathrm{~nm}$, irradiated with 3, 25 or $100 \mathrm{MeV}$ protons. Either the "Livermore" or "Penelope" "Mixed Physics List" is being used denoted as Liv and Pen respectively.

\subsection{Dependence of the dose distribution and dose enhancement from a GNP on selected model}

Figure 6 shows the (A.) RDD and (B.) DER for the selection of models described in Table 1 for a 25 $\mathrm{nm}$ radius GNP irradiated with $25 \mathrm{MeV}$ protons. Again the "(Mixed) Physics List" only affects the 
first $20 \mathrm{~nm}$, and the DER reaches the value of 14 independently of the "(Mixed) Physics List". No influence by the Auger production is observed with the "AugerOff" "(Mixed) Physics List".

(A.)

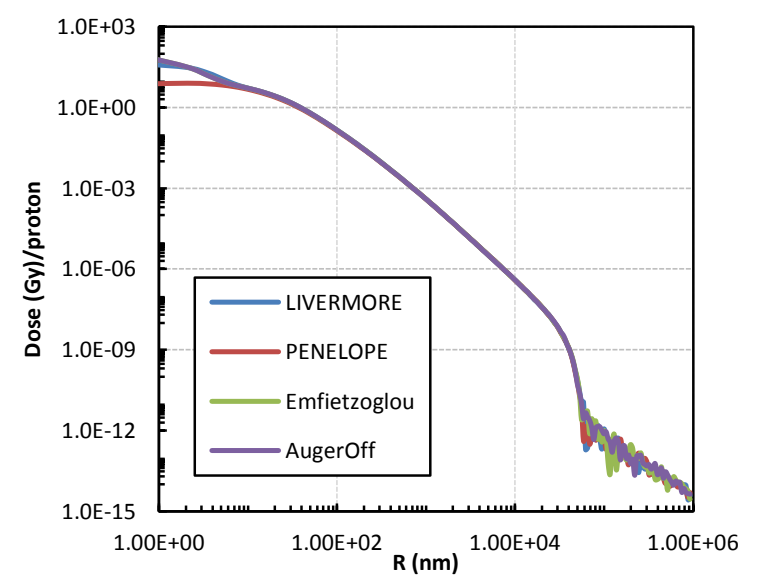

(B.)

DER, 25 nm GNP; 25 MeV protons

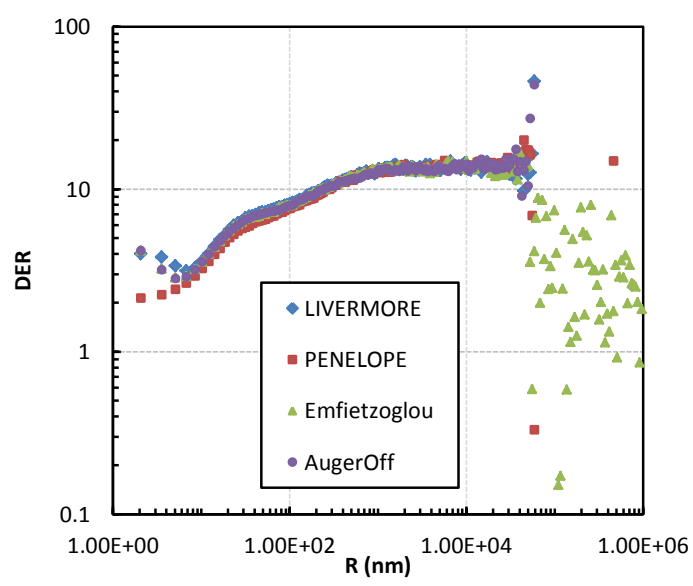

Figure 6. (A.) RDD and (B.) the DER for a GNP with a radius of $25 \mathrm{~nm}$, irradiated with $25 \mathrm{MeV}$ protons. The "Livermore", "Penelope", "Emfietzoglou", or "AugerOff" "Mixed Physics Lists" is used as described in Table 1.

\subsection{Dependence of the dose distribution from a WNP on selected model}

Figure 7 shows the (A.) RDD and (B.) DER for the selection of models described in Table 1 for a 25 nm radius WNP irradiated with $25 \mathrm{MeV}$ protons. The "DNA-Default" case, which is the Geant4-DNA default models in both the WNP and the surrounding water, delivers the highest dose in the close vicinity of the GNP. After $20 \mathrm{~nm}$ all the models result in similar dose deposition (agreement within $5 \%$ ), except the DNA-default where differences of about $20 \%$ are observed with respect to the "Livermore Physics List". The inclusion of the Auger electron production does not affect the RDD (agreement within 5\%) or the DER.

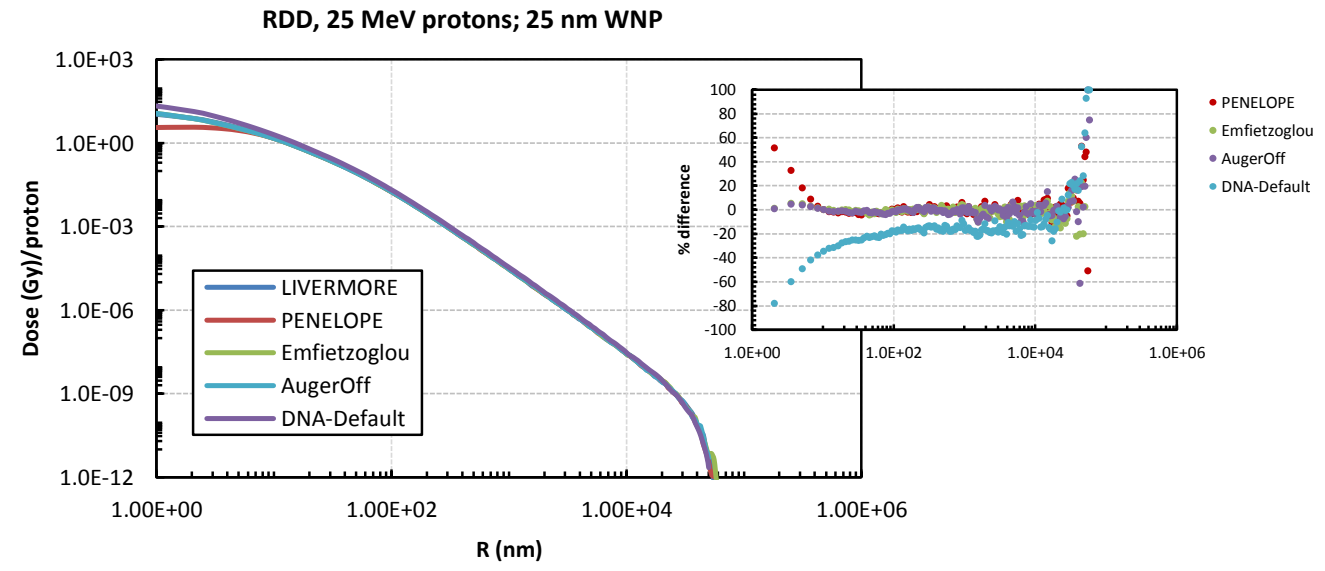

Figure 7. RDD of a WNP with a radius of $25 \mathrm{~nm}$, irradiated with $25 \mathrm{MeV}$ protons. The "Livermore", "Penelope", "Emfietzoglou", "AugerOff" or "DNA-Default" "Mixed Physics List" is used as described in Table 1. The dose percentage difference from the "Livermore Physics List" is also presented.

\subsection{Directionality of the dose distribution and dose enhancement}


Figure 8 presents the impact of the directionality of the proton beam to the (A.) RDD and (B.) DER, for a GNP with the radius of $25 \mathrm{~nm}$ irradiated by $25 \mathrm{MeV}$ protons. The "Livermore Mixed Physics List" is used with the cut-off of 10,100 , and $250 \mathrm{eV}$. The minus sign (-) denotes the direction of the beam, while the plus (+) denotes the opposite direction.
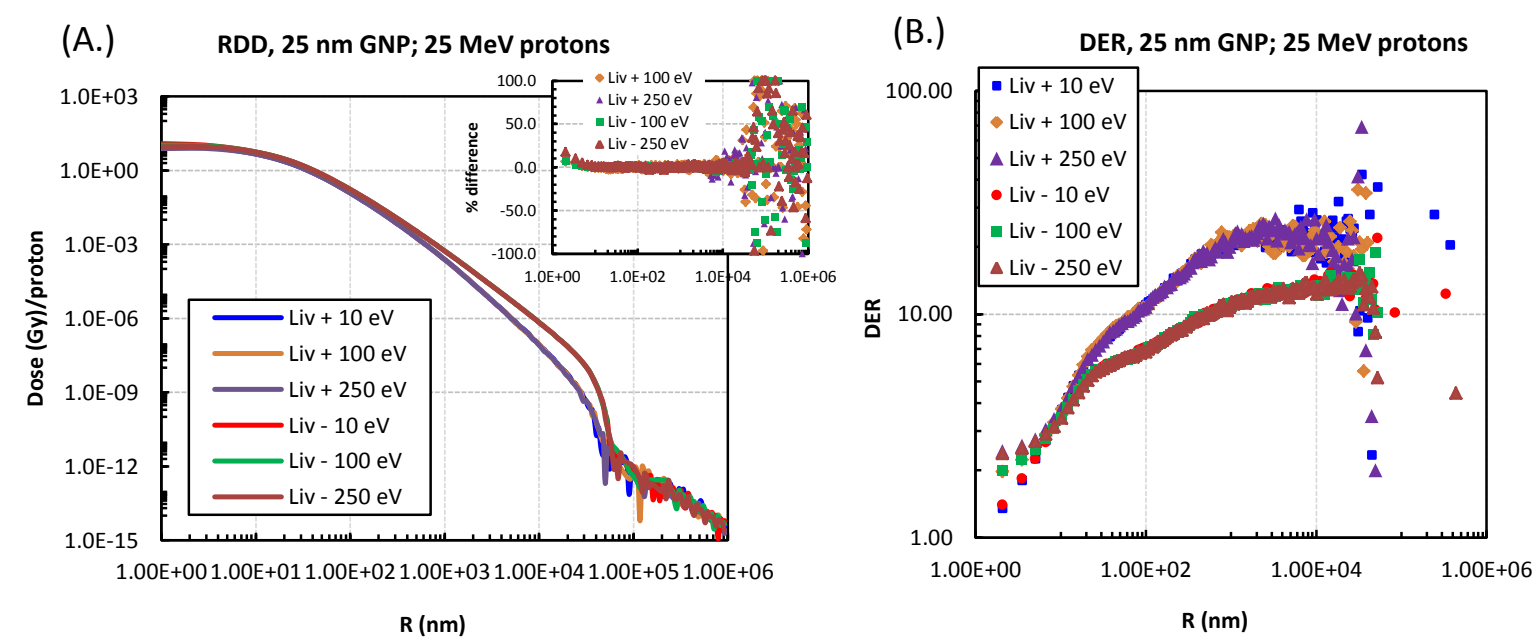

Figure 8. (A.) RDD and (B.) the DER for a GNP with a radius of $25 \mathrm{~nm}$, irradiated with $25 \mathrm{MeV}$ protons. The "Livermore Mixed Physics List" with the cut-off of 10,100, $250 \mathrm{eV}$ is being used, with minus/plus (-/+) sign denoting the beam forward/opposite direction. The dose percentage difference from the "Livermore Mixed Physics List" with the cut-off of $10 \mathrm{eV}$ for each direction is also presented.

\section{Discussion}

The results presented in this work do not demonstrate any practical difference in the radial dose distributions or the dose enhancement ratio by the model combinations (i.e "Mixed Physics Lists"), except if an accurate dose is needed within the first $100 \mathrm{~nm}$ from the GNP surface.

The "Penelope Physics List" has been used in many recent publications, for either calculating the RDD, DER, or in order to create dose distributions to import to a radiobiological model (Leung et al 2011, Lin et al 2015). This selection is based on the original paper presenting the suitability of the "Penelope" models for nanodosimetric (i.e. DNA damage) studies (Bernal and Liendo 2009). On the other hand, simulations based on the "Livermore Physics List" have demonstrated good agreement in reproducing energy spectra of gold nanoparticles or thin foils under photon or proton irradiation (Casta et al 2014, Incerti et al 2015). This work suggests that the choice of the "Physics List" is not important as long as accurate dose estimation close to the GNP is not necessary. It is important to note that the comparison between the "Penelope" and "Livermore" "Physics Lists" is only for the GNP volume. For the water material surrounding the GNP the same Geant4-DNA models are used in all cases.

The difference between the "Livermore" and "Penelope" "Mixed Physics List" in the first $20 \mathrm{~nm}$ may be attributed to the production threshold value. Electrons with energy less than $250 \mathrm{eV}$ will deposit the energy locally. This energy corresponds to approximately $10 \mathrm{~nm}$ range in water, so within this range a reduced dose is expected within the first $10 \mathrm{~nm}$ when compared to the $10 \mathrm{eV}$ cut-off. This effect is reduced with the GNP size, which validates that this reduction is due to the threshold cut-off value and the range of the low energy electrons. Moreover, a limitation in the electron production 
arises from the ionization model. Current Geant 4 proton ionization models cannot simulate the production of $\delta$-rays below the mean ionization energy of the material. However, the same model is used in both cases, and similar influence is expected. The spectrum of the electrons escaping the GNP is presented in Figure 3. A strong dependence to the secondary production cut-off value is observed. Nonetheless, regarding the electron production by the gold material and the implications on the dose deposition, it only influences the production of the low energy electrons that deposit its energy close to the GNP vicinity.

In terms of computational efficiency, the use of the Livermore models with the cut-off value of $10 \mathrm{eV}$ increases the computational time by a factor of about 2. More specifically, the "Livermore", "AugerOff", "Emfietzoglou", and "Penelope" "Mixed Physics List" achieved 3.0, 2.1, 2.5, and 1.4 M particles/hr respectively for the reference case of a $25 \mathrm{~nm}$ GNP irradiated with $25 \mathrm{MeV}$ protons. For the benchmarking an Intel Core i5@2.6 GHz with 8 GB DDR3 RAM running MacOSX 10.11 was used. With the lower cut-off value, a much higher number of low energy electrons is produced inside the GNP and subsequently has to be followed by the much more computationally intensive Geant4DNA models utilized in the surrounding water medium.

The Geant4-DNA models are used for the dose deposition calculations in the water surrounding the GNP. Within the Geant4-DNA framework a selection of different models are available for the dominant processes, especially of ionization and excitation of electrons. Both the default models and the "Emfietzoglou" models for ionization and excitation were investigated with no noticeable difference found. While both are based on the same formalism, the latter are considered more accurate in the low energy regime (Kyriakou et al 2015, 2016). However, the Emfietzoglou models are only applicable for electron energies up to $10 \mathrm{keV}$ with the default models used otherwise, resulting in identical dose distribution.

The "Mixed Physics List" selection for simulating the WNP (shown in Figure 7) has an influence on the calculated RDD, and consequently on the DER. Calculating the dose around the WNP when using the Geant4-DNA default "Physics List" for the interactions inside the WNP results in a higher RDD. When using the "Penelope Physics list", a noticeably lower dose is observed for the same reasons described earlier. While this difference can affect the calculated DER, it is only significant within the first $20 \mathrm{~nm}$.

Electrons produced from proton interactions generated preferentially towards the forward direction. Accordingly to our results in Figure 8 this is true for the high energy electrons (energy greater than 10 $\mathrm{keV}$ ). As a result an increase to the RDD of the hemisphere towards the direction of the beam is observed. In contrast, the DER is higher for the backwards direction. We assume that the backscattering is higher inside the GNP, resulting to a higher DER for the opposite direction.

Another potentially limiting factor of the validity of the current gold models available in Geant 4 for nanoscale simulations is the fact that they are based on condensed history algorithms. Although a good agreement of Geant 4 simulations with experimental data has been demonstrated for spectrum reproduction of thin gold foils or GNPs (Casta et al 2014, Incerti et al 2015), the development of more accurate gold models should improve the secondary electron production. Recently, a discrete electron transport model for Geant4 was developed (Sakata et al 2016) but has not become available in the public release. This improvement is expected to influence the low energy spectrum and consequently the dose deposition in the close vicinity of the GNP.

The experimental setup of this work has been chosen primarily for the evaluation of the Geant4 models available for nanoscale simulation and therefore it has been as simple as possible. McKinnon et al (2016) implemented a more realistic geometry comprising the simulation of the full proton track structure through and outside the NP. The different geometry implemented resulted to different dose enhancement values. On the other hand the spectra of the electrons escaping the GNP generated are very similar. It has to be noted that a simulated spectrum strongly depends on the model selection and 
cut-off values. The inclusion of the atomic de-excitation has only minor effect on the dose enhancement, as also reported by our study.

GNPs are not uniformly distributed into the cell. Clusters of vesicles (a vesicle is a spherical structure resulting from the acquisition process of the GNP from the cell and with typical diameters of $300 \mathrm{~nm}$ ) containing the GNP are formed, localizing the GNPs predominately into the cytoplasm, without entering the cell nucleus (Sadauskas et al 2009, Peckys and De Jonge 2011, Peckys and Jonge 2014, Stefančíková et al 2014). Therefore, the GNPs are away from the nucleus where the DNA is located. In order to achieve a radiosensitization enhancement effect from the GNPs, the increase in the dose has to be significant at a spatial distance of a few $\mu \mathrm{m}$, comparable to the cell nucleus. As the difference between the two models is located within the first $20 \mathrm{~nm}$, no significant implications are expected for the DNA damage and subsequently no impact in the radiosensitization effect.

Although the dose deposition has been extensively used to compare the radiation effects around the GNP, its macroscopic character hides the underlying details of the secondary electron track structure. As long as DNA damage is the main target of the physical component of the GNP radiosensitization, further attention has to be paid to the track structure of the secondary radiation. Recently the nanodosimetry paradigm has been used in order to assess the DNA damage (Grosswendt 2005, Garty et al 2010). Within the nanodosimetry framework the dose deposition profile is not directly considered. Instead the ionisation cluster size distribution is scored in a volume representing a DNA segment of a few base pairs, and then is used to correlate radiation quality to DNA damage. This kind of calculation is more sensitive to the model selection (Lazarakis et al 2012) and further work is planned in order to elucidate the effect of the model selection in the presence of GNPs.

RDDs calculated by Geant 4 Monte Carlo simulations have been utilized in radiobiological models that predict cell survival. More specifically the Local Effect Model (LEM) has been implemented for the calculation of the GNP radiosensitization, demonstrating a good agreement with experimental values (McMahon et al 2011a, McQuaid et al 2016). Current GNP formulations have been shown not to enter the cell nucleus. Under such circumstances the "Mixed Physics List" selected should not have any influence on the final results, as DNA is would be located at too great a distance away from the GNP.

Another case where the "Mixed Physics List" selection might be important is when the free radical production is under consideration. Overall, the free radical production should be more sensitive to the physical interaction model and production threshold used. The initial free radical production is directly proportional to the dose deposition, or equivalently to the secondary electron production (Tran et al 2016). As Tran et al demonstrated, the time evolution of the distribution suggests that the free radicals on average stay in their initial positions. In the case of high free radical density produced in close proximity to the GNP, these free radicals recombine to water reducing the local concentration. As a result, the effect of the "Mixed Physics List" on the RDD is expected to propagate to the free radical production, therefore increasing instantaneous free radical production within the first $20 \mathrm{~nm}$. The new discrete models for the electron transport for gold (Sakata et al 2016) are expected to have the strongest impact on the free radical enhancement.

Considering a more realistic distribution of GNPs within a cell, as described for example in (Peckys and De Jonge 2011, Peckys and Jonge 2014), where vesicles containing the GNP are formed, the "Mixed Physics List" selection should not have any effect because of the multiple contributions of the adjacent GNPs for each point neutralizing the dose enhancement effect.

\section{Conclusion}

The Geant 4 models for the interactions of gold and water with protons were compared in order to investigate whether the "Mixed Physics List" selection may influence the dose or dose enhancement 
around a GNP. It was found that for radial distances larger than $100 \mathrm{~nm}$ from the surface of the GNP no substantial difference should be made by the model combinations of the "Physics Lists" studied. For distances less $100 \mathrm{~nm}$, differences are observed but the relevance depends strongly on the application. Unless more accurate models implementing event-by-event algorithms for the gold interactions with charged particles become available, the dose uncertainty within the first $100 \mathrm{~nm}$ is expected to be high.

The simulated simple irradiation conditions in this paper were selected in the view of establishing a benchmarking geometry that can be later used to compare data from other Monte Carlo codes. An inter-comparison with other codes for nanoscale simulations is envisaged in order to improve the uncertainty estimation in dose deposition calculations of Monte Carlo codes.

\section{Acknowledgements}

This work has been supported by Marie Curie Actions - Initial Training Networks (ITN) as an Integrating Activity Supporting Postgraduate Research with Internships in Industry and Training Excellence (SPRITE) under EC contract no. 317169.

\section{References}

Agostinelli S et al 2003 GEANT4 - A simulation toolkit Nucl. Instruments Methods Phys. Res. Sect. A Accel. Spectrometers, Detect. Assoc. Equip. 506 250-303

Allison J et al 2006 Geant4 developments and applications IEEE Trans. Nucl. Sci. 53 270-8

Bernal M A et al 2015 Track structure modeling in liquid water: A review of the Geant4-DNA very low energy extension of the Geant4 Monte Carlo simulation toolkit Phys. Medica 31 861-74

Bernal M and Liendo J 2009 An investigation on the capabilities of the PENELOPE MC code in nanodosimetry. Med. Phys. 36 620-5

Casta R, Champeaux J-P, Sence M, Moretto-Capelle P, Cafarelli P, Amsellem a. and Sicard-Roselli C 2014 Electronic emission of radio-sensitizing gold nanoparticles under X-ray irradiation: experiment and simulations J. Nanoparticle Res. 162348

Garty G et al 2010 A nanodosimetric model of radiation-induced clustered DNA damage yields. Phys. Med. Biol. 55 761-81

Geant4 Collaboration 2015 Geant4 Physics Reference Manual for the 10.2 release (4 December 2015)

Grosswendt B 2005 Nanodosimetry, from radiation physics to radiation biology Radiat. Prot. Dosimetry 115 1-9

Hainfeld J F, Slatkin D N and Smilowitz H M 2004 The use of gold nanoparticles to enhance radiotherapy in mice. Phys. Med. Biol. 49 N309-15

Incerti $S$ et al 2015 Comparison of experimental proton-induced fluorescence spectra for a selection of thin high-Z samples with Geant4 Monte Carlo simulations Nucl. Instruments Methods Phys. Res. Sect. B Beam Interact. with Mater. Atoms 358 210-22

Incerti $\mathrm{S}$ et al 2010 Comparison of GEANT4 very low energy cross section models with experimental data in water. Med. Phys. 37 4692-708

Incerti $S$ et al 2016 Simulation of Auger electron emission from nanometer-size gold targets using the Geant4 Monte Carlo simulation toolkit Nucl. Instruments Methods Phys. Res. Sect. B Beam Interact. with Mater. Atoms 372 91-101

Kim J-K, Seo S-J, Kim H-T, Kim K-H, Chung M-H, Kim K-R and Ye S-J 2012 Enhanced proton treatment in mouse tumors through proton irradiated nanoradiator effects on metallic nanoparticles. Phys. Med. Biol. 57 8309-23

Kyriakou I, Incerti S and Francis Z 2015 Technical Note: Improvements in geant4 energy-loss model and the effect on low-energy electron transport in liquid water Med. Phys. 42 3870-6

Kyriakou I, Šefl M, Nourry V and Incerti S 2016 The impact of new Geant4-DNA cross section models on electron track structure simulations in liquid water J. Appl. Phys. 119194902

Lazarakis P, Bug M U, Gargioni E, Guatelli S, Rabus H and Rosenfeld a B 2012 Comparison of 
nanodosimetric parameters of track structure calculated by the Monte Carlo codes Geant4-DNA and PTra. Phys. Med. Biol. 57 1231-50

Lechtman E, Mashouf S, Chattopadhyay N, Keller B M, Lai P, Cai Z, Reilly R M and Pignol J-P 2013 A Monte Carlo-based model of gold nanoparticle radiosensitization accounting for increased radiobiological effectiveness. Phys. Med. Biol. 58 3075-87

Leung M K K, Chow J C L, Chithrani B D, Lee M J G, Oms B and Jaffray D a 2011 Irradiation of gold nanoparticles by x-rays: Monte Carlo simulation of dose enhancements and the spatial properties of the secondary electrons production. Med. Phys. 38 624-31

Li W B, Müllner M, Greiter M B, Bissardon C, Xie W Z, Schlatll H, Oeh U, Li J L and Hoeschen C 2014 Monte Carlo simulations of dose enhancement around gold nanoparticles used as X-ray imaging contrast agents and radiosensitizers $903390331 \mathrm{~K}$

Lin Y, McMahon S J, Paganetti H and Schuemann J 2015 Biological modeling of gold nanoparticle enhanced radiotherapy for proton therapy Phys. Med. Biol. $604149-68$

Lin Y, McMahon S J, Scarpelli M, Paganetti H and Schuemann J 2014 Comparing gold nano-particle enhanced radiotherapy with protons, megavoltage photons and kilovoltage photons: a Monte Carlo simulation Phys. Med. Biol. 59 7675-89

Liu C-J et al 2010 Enhancement of cell radiation sensitivity by pegylated gold nanoparticles. Phys. Med. Biol. 55 931-45

McKinnon S, Guatelli S, Incerti S, Ivanchenko V, Konstantinov K, Corde S, Lerch M, Tehei M and Rosenfeld A 2016 Local dose enhancement of proton therapy by ceramic oxide nanoparticles investigated with Geant4 simulations Phys. Medica

McMahon S J et al 2011a Biological consequences of nanoscale energy deposition near irradiated heavy atom nanoparticles Sci. Rep. 11-9

McMahon S J et al 2011b Nanodosimetric effects of gold nanoparticles in megavoltage radiation therapy Radiother. Oncol. $100412-6$

McQuaid H N et al 2016 Imaging and radiation effects of gold nanoparticles in tumour cells Sci. Rep. 619442

Peckys D B and Jonge N De 2014 M icroscopy M icroanalysis Gold Nanoparticle Uptake in Whole Cells in Liquid Examined by Environmental Scanning Electron Microscopy 189-97

Peckys D B and De Jonge N 2011 Visualizing gold nanoparticle uptake in live cells with liquid scanning transmission electron microscopy Nano Lett. 11 1733-8

Polf J C, Bronk L F, Driessen W H P, Arap W, Pasqualini R and Gillin M 2011 Enhanced relative biological effectiveness of proton radiotherapy in tumor cells with internalized gold nanoparticles Appl. Phys. Lett. 98 3-5

Sadauskas E, Jacobsen N R, Danscher G, Stoltenberg M, Vogel U, Larsen A, Kreyling W and Wallin H 2009 Biodistribution of gold nanoparticles in mouse lung following intratracheal instillation. Chem. Cent. J. 316

Sakata D et al 2016 An implementation of discrete electron transport models for gold in the Geant4 simulation toolkit J. Appl. Phys. 120244901

Salvat F, Fernández-Varea J and Sempau J 2011 PENELOPE-2011: A code system for Monte Carlo simulation of electron and photon transport Work. Proc. 384

Stefančíková L et al 2014 Cell localisation of gadolinium-based nanoparticles and related radiosensitising efficacy in glioblastoma cells. Cancer Nanotechnol. 56

Tran H N et al 2016 Geant4 Monte Carlo simulation of absorbed dose and radiolysis yields enhancement from a gold nanoparticle under $\mathrm{MeV}$ proton irradiation Nucl. Instruments Methods Phys. Res. Sect. B Beam Interact. with Mater. Atoms 373 126-39

Wälzlein C, Scifoni E, Krämer M and Durante M 2014 Simulations of dose enhancement for heavy atom nanoparticles irradiated by protons. Phys. Med. Biol. 59 1441-58

Xie W Z, Friedland W, Li W B, Li C Y, Oeh U, Qiu R, Li J L and Hoeschen C 2015 Simulation on the molecular radiosensitization effect of gold nanoparticles in cells irradiated by $\mathrm{x}$-rays Phys. Med. Biol. 60 6195-212 